\title{
PERCEPTUAL LEARNING AS A TOOL FOR BOOSTING WORKING MEMORY AMONG INDIVIDUALS WITH READING AND LEARNING DISABILITY
}

\author{
KAREN BANAI ${ }^{1}$ and MERAV AHISSAR ${ }^{2,3}$ \\ ${ }^{1}$ Department of Communication Disorders, University of Haifa, Haifa, Israel, \\ ${ }^{2}$ Department of Psychology, and ${ }^{3}$ Interdisciplinary Center for Neural Computation, \\ Hebrew University of Jerusalem, Jerusalem, Israel
}

The majority of individuals with dyslexia and additional learning difficulties (D-LDs) also perform poorly on many simple auditory discrimination tasks. We now trained a group of D-LD teenagers on a series of auditory tasks and assessed their pattern of auditory improvement as well as their generalization to reading related tasks. We found that the performance of most D-LD participants quickly improved and reached the level of the general age matched population. Moreover, their pattern of learning specificity (e.g. no transfer from frequency to duration discriminations) was also similar to that previously observed in the general population. When assessed with a battery of verbal tasks that they initially performed poorly, a pattern of specific transfer was observed. Performance on verbal memory tasks improved to peer level, whereas performance on reading and non-verbal cognitive tasks did not. These findings suggest that D-LDs' mechanisms of long-term learning are adequate. Moreover, perceptual learning can be used as a tool for improving general working memory skills, whose underlying mechanisms seem to be shared by simple tones and complex speech sounds.

Keywords: frequency discrimination; duration discrimination; Reverse Hierarchy Theory; auditory memory; auditory processing; dyslexia; learning disability; perceptual learning

* Corresponding author; Department of Communication Disorders, University of Haifa, Mt. Carmel, Haifa 31905, Israel. Phone: +972-4-828-8587; fax: +972+4-824-9507; E-mail: kbanai@research.haifa.ac.il 


\section{INTRODUCTION}

Most individuals with learning disabilities (LDs), also read poorly, and have poor phonological and verbal memory skills (Snow et al., 1998; Everatt et al., 2008). In addition, many LDs also have difficulties with simple auditory discriminations (e.g. Tallal, 1980; De Weirdt, 1988; Reed, 1989; McAnally and Stein, 1996; Heath et al., 1999; Ahissar et al., 2000; McArthur and Bishop, 2001; Witton et al., 2002; Sharma et al., 2006; McArthur et al., 2008). These difficulties led to the formulation of the 'sensory' hypothesis, suggesting that LDs' poorer memory and language abilities stem from poorer stimulus processing skills (Tallal, 1980). This bottom-up account assumes poorer low-level representations of sound features in LDs. An alternative interpretation of these observations is that D-LDs' low-level representations are intact. However, their ability to effectively utilize them is impaired in some contexts. Such an impairment may derive from attentional (Willcutt and Pennington, 2000; Hari and Renvall, 2001; Roach and Hogben, 2008) or working memory (Torgesen, 1988; Swanson, 1993; Swanson and Sachse-Lee, 2001; Gathercole et al., 2006) difficulties that often accompany LD.

Initially we hypothesized that D-LDs' poor performance in simple tasks reflects poor low-level processing (Ahissar et al., 2000). However, in a series of subsequent studies (Ben-Yehudah et al., 2001; Amitay et al., 2002a; Amitay et al., 2002b; Banai and Ahissar, 2004; Ahissar et al., 2006; Banai and Ahissar, 2006) we found that their difficulties depend on task protocol more than on the complexity of stimulus or the perceptual resolution required. Specifically, while the general population performs substantially better when a limited set of stimuli is used repeatedly, individuals with D-LD display impaired 'anchoring' ability, and their thresholds remain high even with stimulus specific repetitions (Ahissar et al., 2006). Such an anchoring deficit may limit the accessibility to "best low-level representations" (Ahissar, 2007). Thus, while in the general population, repeated stimuli enable access to a selective low-level population which is repeatedly activated across trials (Reverse Hierarchy Theory, RHT, Ahissar and Hochstein, 1997, 2004), this enabling process seems impaired among many D-LDs. According to RHT, the fine thresholds revealed in the general population when a small set of stimuli is presented repeatedly, reflect low-level resolution. Among D-LDs such protocols may not reveal low-level resolution since the repeated stimuli cannot be anchored to and hence do not facilitate such access (Nahum et al., 2008).

Many studies demonstrated substantial learning effects in adult performance of simple tasks (see Goldstone, 1998; Fahle and Poggio, 2002; Fahle, 2005), suggesting that perceptual representations remain plastic throughout life. Yet, only little is known about perceptual learning in dyslexia. Two studies suggest a large degree of improvement, and substantial transfer from several weeks' training on simple discriminations (intensity and frequency discrimination, gap detection, temporal order judgment and lateralization) (Schaffler et al., 2004), or on audio-visual pattern matching (Kujala et al., 2001) to spelling and reading, respectively. On the other hand, no transfer of learning from perceptual to reading related skills occurred in a third study in which listeners trained (and improved) on a single perceptual discrimination task (either frequency, vowel or consonant-vowel discrimination or rapid auditory processing) (McArthur et al., 2008). 
In the current study we trained listeners on a series of auditory discrimination tasks and compared the dynamics of learning and generalization to the patterns previously found in the general population. We also tested generalization to cognitive, reading and memory skills, to determine whether specific links between perception and those skills exist. We hypothesized that: (1) If poor performance stems from a common high level factor such as a general lack of listening skills (poor auditory attention), improvement is expected to transfer across dimensions. (2) If learning either improves access to low-level representations, or improves the accuracy of low-level representations themselves, it is expected to show similar learning dynamics as those found in the general population, since both aspects probably take place in perceptual learning in the general population. (3) If training only involves improved low-level accuracy it is not expected to transfer to tasks that are not limited by low-level resolution. Thus, it is not expected to transfer to improved memory of easily perceived and discriminated items. On the other hand, if anchoring is improved, and this improvement generalizes beyond the trained stimuli, working memory skills may improve even for untrained stimulus types.

\section{METHODS}

\section{Participants}

Twelve female $8^{\text {th }}$ grade students (ages $14.2 \pm 0.4$ ) with reading and additional learning disabilities $(D-L D s)$ were trained as a part of a larger study on the core perceptual deficit in dyslexia (Ahissar et al., 2006; Banai and Ahissar, 2006). From the larger group of participants (a class in an all girl school for individuals with mild learning difficulties) we selected the 12 students with the poorest frequency discrimination abilities (see below) for training. Because selection based on frequency discrimination resulted in a group that was nearly identical to the groups of poor readers with additional learning disabilities (D-LD) described in our previous studies, we use the term here as well. The other students from the same class (peers, $\mathrm{n}=10$ ) had normal frequency discrimination and normal reading scores (see Banai and Ahissar, 2006, study I) and were therefore not trained. All students (trained and untrained) were tested on frequency discrimination, reading, cognitive and memory measures 3 times: before and after the training period and 5 months later. The inclusion of the untrained peers served to determine benchmark performance for this age group during the pre- and post-training assessment periods (which spanned $\sim 10$ months). It also allowed us to estimate whether there are substantial changes associated with re-measuring those skills several months apart in this age group. Additional comparisons of the data from the trained group were made to data of naïve D-LDs from our previous studies, whose initial frequency discrimination scores were similar (Banai and Ahissar 2006, study II and Ahissar et al., 2006). The study was approved by the ethics committee of the Department of Psychology at the Hebrew University of Jerusalem, and informed consent was obtained from the parents prior to the study. 


\section{Procedure}

The experiment was conducted in 4 phases: 1) pre-test; 2) training (administered to D-LDs only); 3) an immediate post-test 4 ) a second post-test, conducted 5 months later.

Training consisted of a series of tone discrimination tasks, beginning with frequency discrimination and continuing with duration discrimination. The introduction of novel tasks was contingent upon reaching peer level performance scores. Hence, not all participants completed the full series of tasks (see below). Using this protocol allowed us to characterize the specificity/generalization pattern of tone discrimination learning to other discrimination tasks along the same dimension (new parameters) and across dimensions. It further allowed us to assess the impact of the whole training procedure on verbal scores. However, it did not allow us to evaluate at which stage of practice generalization to verbal tasks began to emerge (when such generalization was observed).

\section{Pre- and post-training assessments}

Auditory frequency discrimination (FD). Two $50 \mathrm{~ms}$ tones with $1000 \mathrm{~ms}$ inter-stimulus interval (ISI) were presented in each trial with a standard reference tone of $1000 \mathrm{~Hz}$ and a non-standard (presented either first or second) which was always higher. Listeners had to indicate which tone was higher $\left(1^{\text {st }}\right.$ or $\left.2^{\text {nd }}\right)$. (See Banai and Ahissar, 2006 for further details.) Thresholds were measured twice before training, once at post-test I and twice at post-test II.

Reading. The speed and accuracy of reading single Hebrew words and non-words was tested using lists of 24 items designed by A. Deutsch (Deutsch and Bentin, 1996).

Phonological awareness was assessed using the Hebrew version of the Spoonerism task (developed by Peleg and Ben-Dror). Listeners were asked to swap the first phonemes of two words presented orally by the tester (e.g. white pig $\rightarrow$ pite wig). Twenty pairs were presented.

Memory. Both span and working memory were assessed using: (1) Digit Span Forward (span) and Backwards (working memory) (Wechsler, 1998). (2) Word Span for common Hebrew words. (3) Working Memory Span (Designed by Ben-Dror and Shany). This test requires listeners to generate the opposites of common Hebrew adjectives in order of presentation.

Speech perception. Stimuli were pairs of 24 pairs of disyllabic pseudo-words differing in one consonant, using the following minimal contrast pairs: d-t, b-d, b-p, v-f, m-n, s-z (each presented twice). Twenty-four different pairs were used. In each trial, a pair of pseudo-words was played. After a $2 \mathrm{~s}$ interval, one stimulus was repeated and listeners had to indicate whether it was the $1^{\text {st }}$ or the $2^{\text {nd }}$ (see Banai and Ahissar, 2006 for details). Due to the near perfect performance of Controls in the pre-test, they were not tested again on this task in the post-test.

General cognitive abilities. (1) Block Design (Wechsler, 1998) was used as a measure of general, non-verbal, cognitive abilities. (2) Raven's Standard Progressive Matrices (Raven et al., 2000). All participating students had scores of 35 or higher, which is $>25^{\text {th }}$ percentile (equivalent to I.Q. scores $>80$ ). We administered this test again as part of post-test II. 


\section{Training}

Trained participants practiced for 7 weeks (28-32 training sessions per student, typically four sessions every week). In each session participants completed 1-4 training blocks (typically 2-3), each lasting 100 trials or 20 reversals, whichever was attained first. A typical daily session lasted 20 minutes. Thresholds were determined as the average of the last 10 reversals of each block.

All training tasks were 2-alternative forced-choice tasks. On each trial, a fixed standard tone and an adaptive test tone were played with a $1000 \mathrm{~ms}$ inter-stimulus-interval (ISI), and the listener had to determine the interval in which the test tone was played. The test tone was adapted based on subjects' performance using the 2 down/1 up staircase procedure. Both positive and negative feedback were provided in the form of a smiling and a sad face, respectively. At the end of each training block participants watched a short funny, silent, video clip on the computer screen before continuing to the next block. Training on a given task lasted until criterion level of average peer performance was reached.

To maintain the motivation for practice, we adopted a 'points' system. Participants could trade the points they accumulated for small prizes (e.g. mugs, pens) at the end of every training week, or for larger prizes (e.g. CDs) at the end of the whole training program. They were also given a nice sticker for each block they completed.

\section{Frequency discrimination (FD)}

The first task trained was frequency discrimination (FD) with a $1000 \mathrm{~Hz}$ fixed reference tone. As in the pre-training assessments, each of the 2 subsequently presented tones was $50 \mathrm{~ms}$ long. Listeners had to decide which tone was higher. Each student kept training on this task until she reached a threshold $\leq 15 \%$ (peers' average pre-training threshold). After reaching criterion, one session was devoted to testing the same task with $500 \mathrm{~Hz}$ and $2000 \mathrm{~Hz}$ reference tones to assess transfer across octaves. Over the entire training period, participants trained on frequency discrimination for 4-10 sessions (1200-3000 trials), depending on individual learning rate.

Immediately before the first post-test, trained listeners were tested on a similar FD task, but without a repeated standard (FD-No-Standard; see Ahissar et al., 2006).

\section{Duration discrimination}

Participants were asked which of two $1000 \mathrm{~Hz}$ tones was longer. Practice began using a 100 $\mathrm{ms}$ long standard for 1-3 training sessions, followed by 1-2 sessions of training with $200 \mathrm{~ms}$ standards and 1-2 sessions of training with $400 \mathrm{~ms}$ standards. Subsequently, participants were trained with $100 \mathrm{~ms}$ standards for 1-2 additional sessions. Over the entire training period, participants practiced duration discrimination for 4-8 training sessions (1200-2400 trials). Baseline levels around 100 and $400 \mathrm{~ms}$ for naïve peers and naïve D-LDs with similar frequency discrimination performance were obtained in a previous study (Banai and Ahissar, 2006) and were used here for comparison. 
Additional tone discrimination tasks

Participants who reached peer level performance on the 2 tasks described above continued practicing the tasks described below until the end of the training period.

AM detection - test tones were $1000 \mathrm{~Hz}, 1$-s' carriers modulated at 4, 8, 10 or $16 \mathrm{~Hz}$ (in separate blocks). Subjects performed this task for 3 sessions, and 2-4 additional sessions with shorter $(250 \mathrm{~ms})$ stimuli. Eight listeners reached this stage.

AM Rate Discrimination - A fixed standard (1-Khz, 1-s tone, modulated at 2, 4 or $8 \mathrm{~Hz}$ in different blocks) had to be discriminated from a test tone modulated at a slightly higher rate. Only 3 listeners performed this task.

Re-assessments of frequency discrimination

Towards the end of training, students were re-assessed with the original FD-Standard task. ISI was then gradually decreased to $600,400,200$ and $100 \mathrm{~ms}$ in separate subsequent assessments. Two-three blocks were measured for each ISI. The first block was used to assess generalization from the trained $1000 \mathrm{~ms}$ ISI to the untrained ISI. Subsequent block/s provided further training.

The final $\left(8^{\text {th }}\right)$ week of training comprised of re-administration of the FD-Standard task with $1000 \mathrm{~ms}$ ISI with 500 and $1000 \mathrm{~Hz}$ reference tones, duration discrimination with 100 and $200 \mathrm{~ms}$ reference tones, $4 \mathrm{~Hz}$ AM detection and AM rate discrimination with $4 \mathrm{~Hz}$ reference. In addition, performance on the FD-No-Standard task was assessed. One threshold was measured for each of these conditions. Post-test I was conducted the following week.

\section{RESULTS}

\section{Pre-training screening}

The poor frequency discrimination performers were all poor readers and had poor language scores (hence the term D-LDs; Table 1, pre-training) compared with their class peers. D-LDs' general cognitive scores, though within the normal range, were also somewhat lower than those of their peers.

\section{A summary of frequency discrimination (FD) learning}

Training improved FD-Standard thresholds of 10/12 D-LD participants to the level of their untrained normal-reading peers (see Figure 1A, pre-test vs. post-tests). Improvement was 


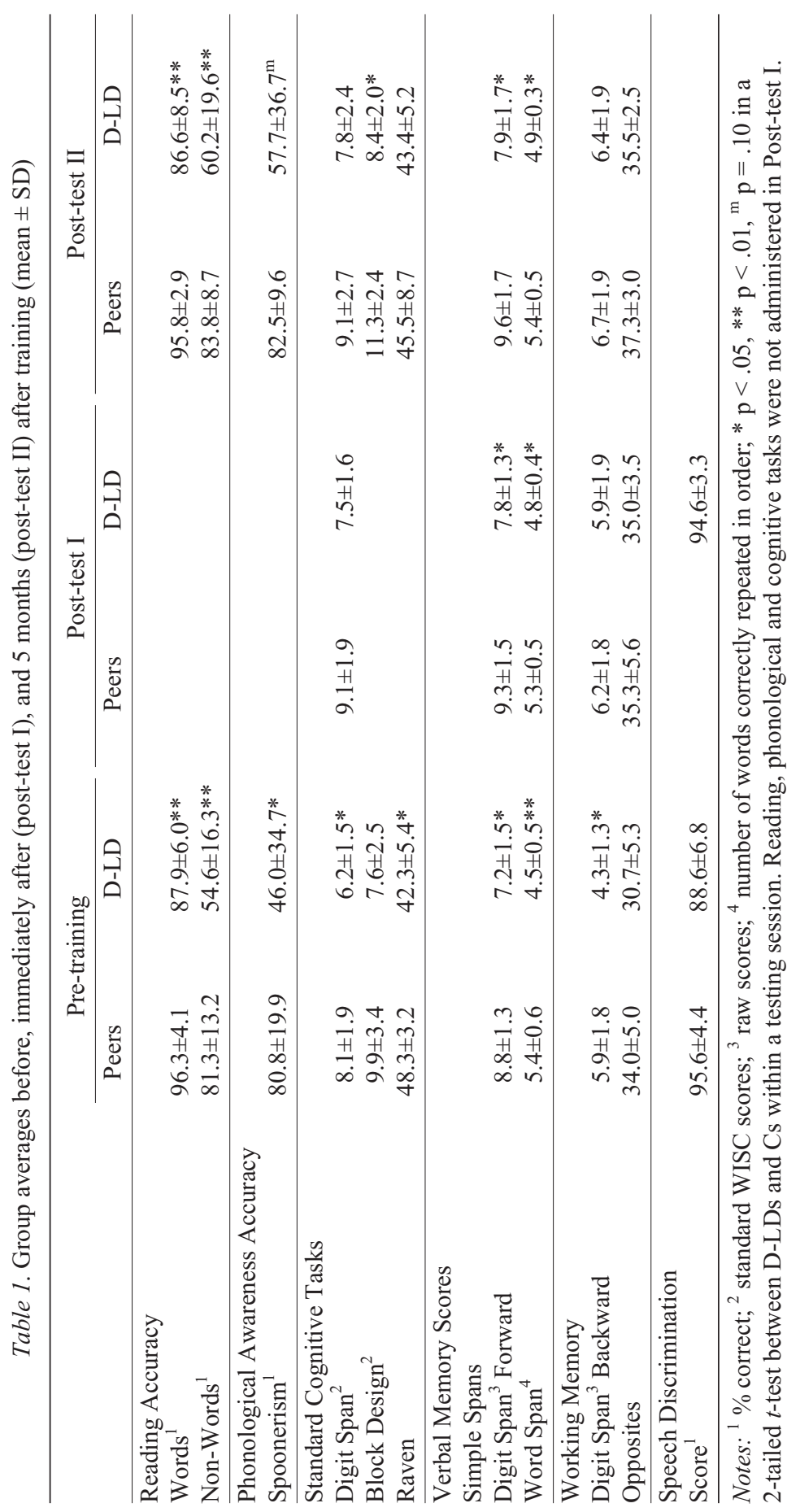

Learning \& Perception 1 (2009) 1 
maintained for at least 5 months after the end of training, when a $2^{\text {nd }}$ post-test was administered. A 3 (time: pre-training, post-test I, post-test II) $\times 2$ (group: D-LDs vs. peers) Repeated Measures ANOVA revealed significant effects for both group $(\mathrm{F}=18.49, \mathrm{p}<.001)$ and time of measurement $(\mathrm{F}=22.8, \mathrm{p}<.001)$ and a significant group $\times$ time interaction $(\mathrm{F}=13.48, \mathrm{p}=$ .002 ), indicating that the thresholds of the trained D-LDs improved significantly more than those of their peers, that is, the improvement expected by test-re-test effects and normal development.

No changes in thresholds occurred between the two post-test sessions in either group (planned comparisons, $\mathrm{p}>0.18$ ). Thresholds of the peers remained stable across the three assessments indicating that repeated test sessions administered months apart do not result in significant learning.

\section{Dynamics of improvement on FD}

D-LDs' learning curve (averaged for 10/12 participants, see below), on the FD-standard task is shown in Figure 1A. Overall improvement rate of most participants was quite fast, however it greatly varied between individuals. Thus, by the end of the $2^{\text {nd }}$ training session, 7 participants reached criterion-level performance (15\%), and consequently began training on the next task. The figure shows only the first 3 training sessions, since only those were administered to all participants. Figure $1 B$ illustrates the learning curve of one of the seven quick learners. The $8^{\text {th }}$ participant reached criterion in the next session. The remaining four (we began with 12) did not show any sign of improvement throughout these 4 sessions, and we there-

\section{Notes to Figure 1}

A. Average learning curve. From left to right: The two pretest assessments of the trained D-LDs (filled symbols) and their peers (empty symbols; see Table 1), thresholds measured in 3 subsequent assessments, crit - average Hi/Lo JND in the last assessment during the training period. freq - JNDs measured around 500 and $2000 \mathrm{~Hz}$ reference tones, after criterion level was reached for $1000 \mathrm{~Hz}$. Nearly complete transfer was found for $2000 \mathrm{~Hz}$, and somewhat less so for $500 \mathrm{~Hz}, I S I$ - JNDs measured in the subsequent session using a $1000 \mathrm{~Hz}$ reference tone with 400, 200 and $100 \mathrm{~ms}$ ISIs. Complete transfer was found, except for the shortest, $100 \mathrm{~ms}$ ISI. end training - JNDs measured at the end of the 8 week training period. Post-tests - JNDs assessed in an immediate and in a 5 months delayed post-test for D-LDs and peers. Error bars indicate \pm 1 s.e.m. B, C, D. Individual examples. B. A learning curve of a fast learner. C. A slow learner who was administered longer tones to "boost" learning. Open squares and pentangulares indicate blocks with 250 and $150 \mathrm{~ms}$ tones, respectively. Note that after two successful practice sessions with long tones, performance with short tones was still poor (session 9). However, following subsequent practice with $150 \mathrm{~ms}$ tones, improvement transferred to brief tones (crit). Both subjects showed complete generalization to shorter ISIs, nearly complete transfer to an octave above the trained frequency and less transfer to one octave below the trained frequency. D. A Non-learner. Success rate on a same/different task with tones separated by one (grey bars) or two (black bars) octaves. Even after extended practice this student had difficulties performing the task when the frequency difference between the tones was one octave or when tones were brief. The error bars, plotted for the conditions she performed 3 times or more ( 50 and $150 \mathrm{~ms}: \mathrm{n}=3,200 \mathrm{~ms}: \mathrm{n}=6)$, show that her performance was consistently adequate with the longer tones and consistently near chance level with brief tones. 
A

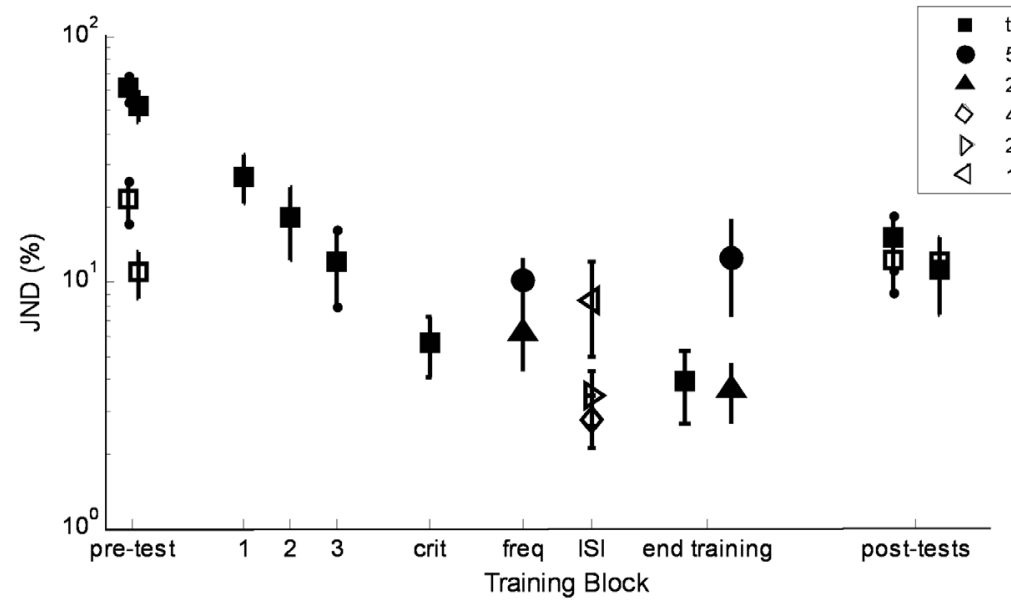

B Fast Learner

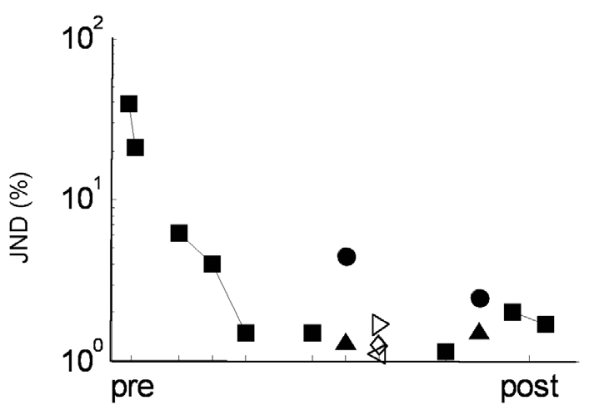

C Slow Learner

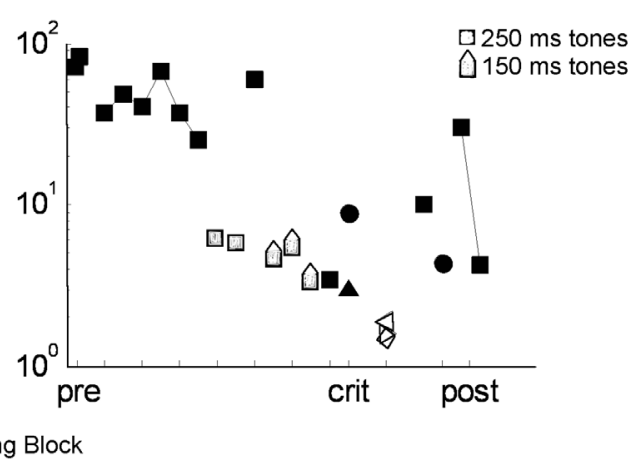

D Non Learner

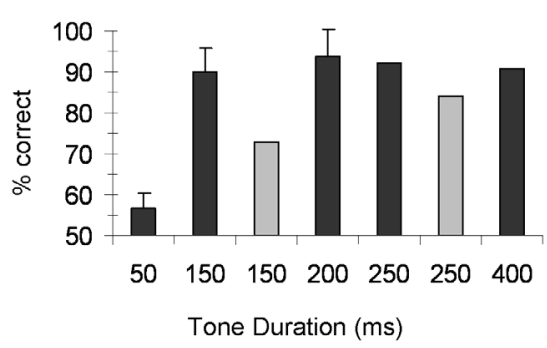

- Tones separated by two octaves $\square$ Tones separated by 1 octave

Figure 1. Learning and generalization of frequency discrimination. 
fore tried to facilitate their performance by delivering longer, $250 \mathrm{~ms}$ tones. This elongation induced significant improvement for 2 of them. As shown in the Figure $1 C$ (for one of these participants), her performance improved immediately upon the introduction of longer tones, but transfer to $50 \mathrm{~ms}$ tones was not immediate and required 3 more practice blocks with intermediate $(150 \mathrm{~ms})$ tone durations. The other participant reached criterion only after a very long training process ( 35 blocks) which spanned the full duration of the training program. She thus never got to practice other tasks. Because she missed many school days, it is possible that her practice sessions were too far apart for learning to consolidate.

Together, these data show that 3-10 training blocks, i.e. 300-1000 trials, were sufficient to improve FD thresholds for the majority of D-LDs to average peer-level performance, suggesting that their initially impaired performance did not stem from a comprehensive low-level deficit in auditory processing. The gradual improvement shown in Figure 1 further suggests that, in line with earlier studies of D-LDs, the pre- to post-training improvement in FD is the result several training sessions rather than the re-test itself.

\section{Non-learners}

Two of the trained subjects never showed any improvement even though they kept practicing throughout the training period, and even though we tried $0.5 \mathrm{~s}$ long stimuli in attempt to facilitate their performance. One of the two had the poorest working memory and general cognitive skills in our sample. That is, she could not repeat correctly more than two digits in reverse order. The other non-learner had normal non-verbal cognitive skills (i.e. Block Design score), but her Digit Backwards score (3) was low (though 6 other D-LDs, who managed to improve on the FD task also scored 3).

Another characteristic of these two students was that they could not perform adequately even on a same/different frequency discrimination task, which the rest of the D-LDs had no difficulty with (see also Banai and Ahissar 2006). Both had a success rate $<70 \%$ on the same/different task when measured with $50 \mathrm{~ms}$ tones. Therefore, part of their training procedure was a same/different task with longer tones, spaced more than one octave apart. One of the two (with poorest memory scores), could perform the same/different task under these conditions, as shown in Figure 1D. However, her performance with brief tones never improved much above chance level. Furthermore, even with longer tones, she could never master the high/low discrimination even though she seemed to understand the task when demonstrated on a piano in the schools' music room. The other non-learner, managed to learn the same/different task, and even seemed to understand the high/low task with long tones ( $>200 \mathrm{~ms})$. However, even with long tones, she could not perform this task consistently for more than 20 or 30 trails, precluding her from obtaining reasonable thresholds.

Since these 2 individuals could not master even the first, FD task, their data were discarded from further analysis and are not shown on any figure. 


\section{Transfer within the domain of simple tone discriminations}

\section{Transfer to new reference frequencies and inter-stimulus intervals}

After reaching the criterion threshold on FD around the trained $1000 \mathrm{~Hz}$ standard tone, students were tested with the same protocol but with two new standards $(2000$ and $500 \mathrm{~Hz}$, measured in that order). Their pre-training performance on these standards was not measured. Based on prior assessments with naïve D-LDs (Banai and Ahissar, 2004) we assumed it was impaired. We now found that their measured thresholds around both references did not significantly differ from those attained for the trained $1000 \mathrm{~Hz}$ (Fig. 1A, triangles and circles, respectively, JND = 5.71 $\pm 5.0,10.10 \pm 7.4,6.20 \pm 5.4$ for the 1000,500 and $2000 \mathrm{~Hz}$ conditions, respectively; $\mathrm{F}=1.41, \mathrm{~ns})$, and were significantly better than its pre-training thresholds $(\mathrm{F}=$ $9.34, \mathrm{p}=0.01)$. Thus, there was a significant generalization to untrained frequencies an octave apart from the trained one. While not statistically different, it seems that there was complete generalization to $2000 \mathrm{~Hz}$ and perhaps only partial generalization to $500 \mathrm{~Hz}$.

Performance with shorter ISIs was also expected to be initially impaired (Ben-Yehudah et al., 2004). Yet, after training with $1000 \mathrm{~ms}$ ISI, trained D-LD performance with shorter intervals was adequate (Fig. 1A, symbols plotted above the ISI mark). Their thresholds with shorter ISIs were similar to those reached with the trained interval $(F=1.9 ; \mathrm{p}=.17)$ and were significantly lower than the threshold obtained initially (the first block of the first training session) with $1000 \mathrm{~ms}$ ISI $(\mathrm{F}=10.8 ; \mathrm{p}<.001)$.

\section{Transfer and learning of duration discrimination}

D-LDs' performance on the first block of duration discrimination with 100 -ms reference tones, indicates that frequency discrimination learning did not generalize to duration discrimination (Fig. 2A). Their thresholds were not significantly different from those of naïve D-LDs $(\mathrm{t}=0.84, \mathrm{p}=0.4)$, and were significantly worse than those of naïve peers $(\mathrm{t}=2.35, \mathrm{p}=0.038$; naïve and peer data taken from Banai and Ahissar, 2006), as shown in Figure 2 (compare filled symbols of trained D-LDs to open circles and open diamonds denoting naïve D-LDs and adequately-reading peers, respectively).

Following few (1-4) training sessions with 100-ms reference, subjects trained for 1-2 sessions with $200 \mathrm{~ms}$, and then for 1-2 more sessions with $400 \mathrm{~ms}$. Afterwards subjects had few more training sessions with $100 \mathrm{~ms}$ reference. Learning curves varied between subjects, as shown in the examples of Figure $2 \mathrm{~B}$. Overall performance around $100 \mathrm{~ms}$ tended to improve (first vs. last block: $50.4 \pm 26$ and $30 \pm 23 \mathrm{~ms}$, respectively; $\mathrm{t}=1.67, \mathrm{p}=0.065$, see Figure $2 B$ for individual examples), and reached peer level performance ( $30 \pm 23$ vs. $28 \pm 13, \mathrm{t}=0.24, \mathrm{p}>$ 0.8). Participants' initial thresholds with $200 \mathrm{~ms}(24.5 \pm 11 \%)$ were similar to those reported in the general population (Wright et al., 1997; Karmarkar and Buonomano, 2003) suggesting that the effects of practice with $100 \mathrm{~ms}$ transferred to $200 \mathrm{~ms}$ tones. On the other hand, there was no generalization to the 400-ms condition; thresholds of trained D-LDs were significantly different from those of unimpaired peers and were very similar to those of naïve D-LDs (illustrated in Figure 2A). 

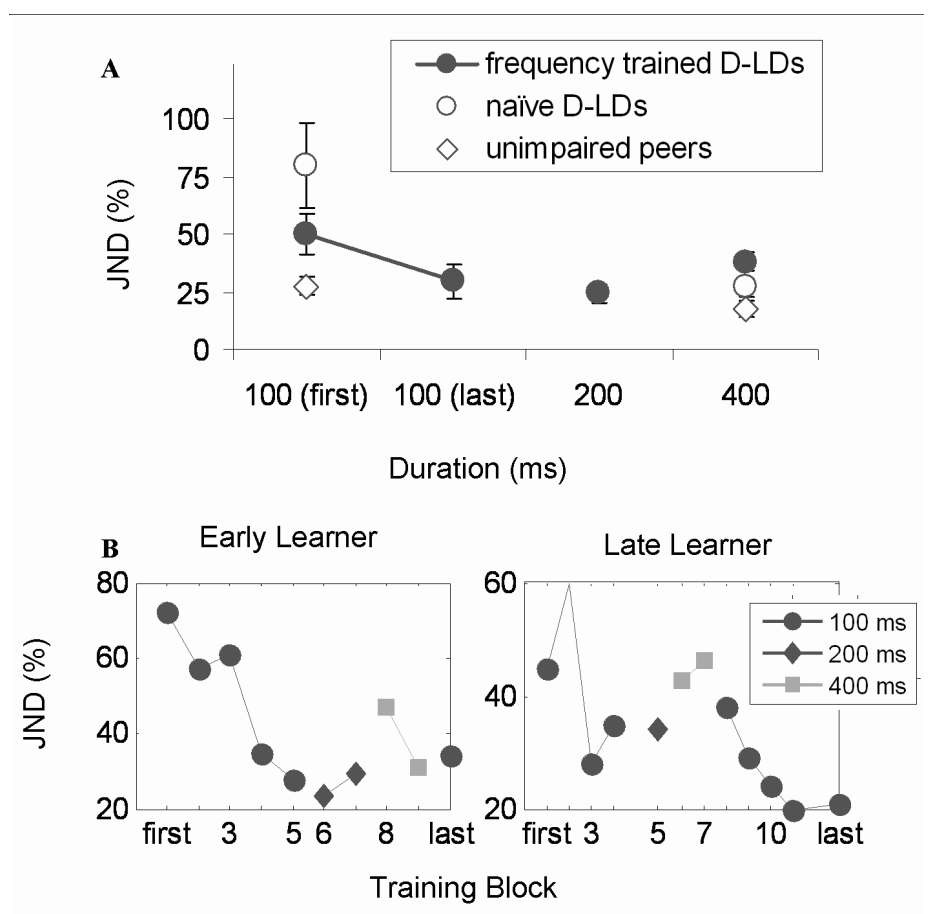

Figure 2. Duration discrimination learning and performance curves. A. Average performance. JNDs of trained D-LDs are shown for the first and last assessment around $100 \mathrm{~ms}$ and for the first blocks measured with 200 and with $400 \mathrm{~ms}$ tones. Data from untrained D-LDs and unimpaired peers (taken from Banai and Ahissar, 2006) is shown for comparison. B. Examples of two individual learning curves. Curves demonstrate different patterns of learning observed among participants - an early learner (left) improved most on the first five blocks of practice whereas a late learner (right) still learned much after the $6^{\text {th }}$ block of practice.

\section{Transfer to the no-reference condition}

Towards the end of the training period, thresholds for FD were assessed using a no-reference condition. The measured thresholds, $15.7 \pm 8 \%$, were $\sim 3$ times higher than those in the reference containing condition (a highly significant difference $t=3.5, p<.01$ in a paired comparison). This ratio, illustrated in Figure 3 (filled circles) is similar to that observed in the general population, which greatly benefit from using a repeated reference across trials compared with a similar paradigm which does not include a reference. On the other hand, naïve D-LDs do not benefit from stimulus specific repetition (Figure 3, empty circles, data taken from Ahissar et al., 2006). Taken together, these data suggest that the training procedure increased the anchoring ability (benefit from reference repetition) among D-LDs to the level of their unimpaired peers, at least in the context of frequency discrimination. 


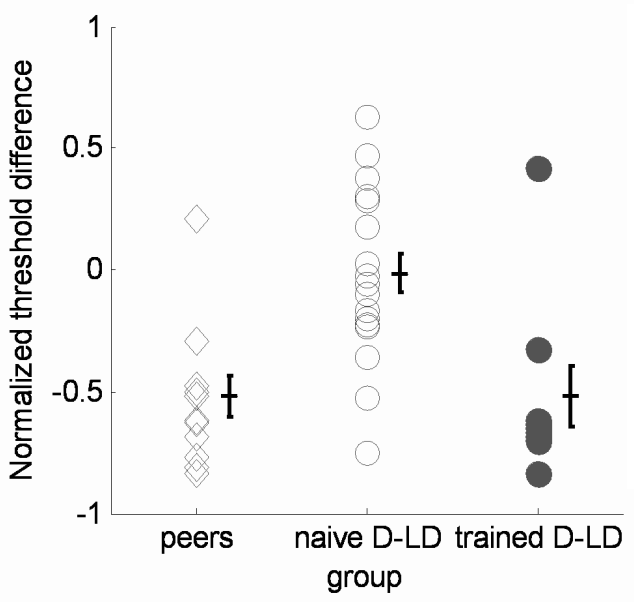

Figure 3. Ratio between FD thresholds with and without a repeated reference (anchoring score). Single subject data on the normalized threshold difference $($ NTD $=($ Standard - No Standard $) /($ Standard + No Standard) for 3 populations. Unimpaired peers (right, diamonds), naïve D-LDs (empty circles, taken from Ahissar et al., 2006), and the trained D-LDs. While naïve D-LDs do not benefit from the reference condition $(\mathrm{NTD}=0)$ trained D-LDs reach benefit levels of their unimpaired peers. The negative values indicate that thresholds with reference were smaller, i.e. better, than thresholds measured in the no reference condition; Error bars indicate \pm 1 SEM.

\section{Transfer to a discrimination task using speech stimuli}

All training tasks shared two basic properties. First, only simple tones were used. Second, all thresholds were assessed using the same reference containing behavioral task, and the same adaptive staircase procedure. We now asked whether improvement transferred to tasks in which stimuli were complex speech sounds and task structure was different. The first task we assessed was speech memorization and comparison. Pre-training D-LDs' performance was less accurate than that of their unimpaired peers (see Table $1 ; \mathrm{t}=2.59, \mathrm{p}=.02$ ). After training, their accuracy on this task significantly improved $(\mathrm{t}=2.66, \mathrm{p}=.028)$ and reached a similar level to that of their peers. Yet, because we have not measured performance on this task twice in any other group, we cannot rule out the possibility that this improvement resulted from a test-re-test effect.

\section{Transfer to memory tasks}

Average Digit Forward span did not significantly improve in the trained group from pre- to post-test, and as shown in Table 1, significant group differences were found both before and after training. However, the number of digits D-LDs could repeat correctly in reverse order (Digit Backwards) substantially increased, from $3.7 \pm 1.06$ to $4.7 \pm 1.14$ so that post-test scores did not significantly differ between D-LDs and their peers (Fig. 4A). A significant ef- 
A.

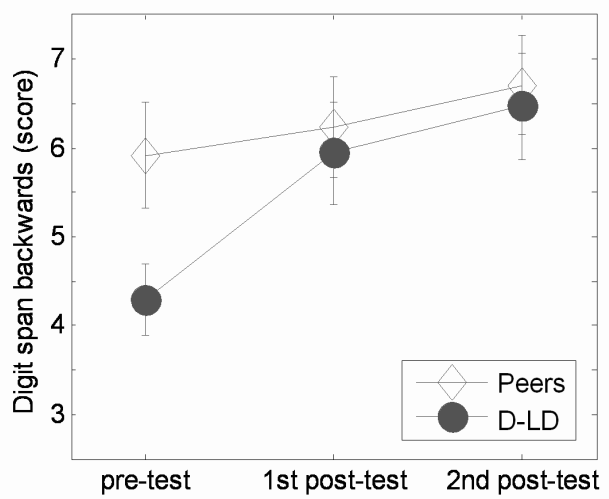

B.

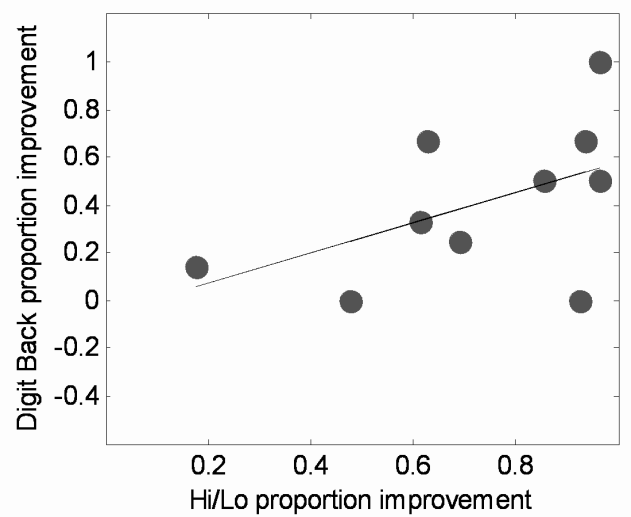

Figure 4. A. Working memory scores before, at the end, and 5 months after training. Average Digit Backward scores of D-LDs and their unimpaired peers are shown, for these 3 assessments. Error bars indicate \pm 1 SEM. B. D-LD's improvement in working memory versus their improvement in frequency discrimination. Correlation between proportion improvement in Digit Span Backward (data from post-test I) and proportion improvement in FD. Proportion improvement was calculated as the difference between the pre- and post-test values divided by the pre-test value. The black line denotes the linear fit.

fect for time $(\mathrm{F}=8.7, \mathrm{p}=.01)$ and a significant time $\times$ group interaction between the pre-test and the first post-test $(\mathrm{F}=4.8, \mathrm{p}=.04)$, indicate that $\mathrm{D}$-LDs improved significantly more than unimpaired peers between the two assessments. This improvement was retained for at least 5 months, as found in the second post-test.

A similar trend was found for word span (see Table 1). Forward span did not significantly improve with training. Yet, a working memory span task, which required the transformation of a list of adjectives to the list of their opposites, significantly improved between preand post-test in the trained group $(\mathrm{t}=2.6, \mathrm{p}=.031)$, but not among unimpaired peers $(\mathrm{t}=.9$, $\mathrm{p}>.4)$. 
Taken together, training significantly improved performance on tasks relying on active working memory (i.e. serial comparisons of pseudo-words, Digit Backwards, word opposites), whereas it only slightly improved simple spans. The 2 post-tests were statistically indistinguishable, indicating no decay of the learning effects. Furthermore, as shown in Figure $4 B$, the amount of improvement in working memory among D-LDs tended to correlate (i.e. the correlation was marginally significant, Spearman rho $=.59, \mathrm{p}=.07$ ) with the amount of training related improvement in frequency discrimination. This was not the case among peers, in which no relationship was found (Spearman rho $=-.07, \mathrm{p}>.8$ ). These findings further support the suggestion that the improvement in working memory scores is specifically related to the 2-tone discrimination training.

\section{Reading, phonological and cognitive tasks}

Though training improved working memory scores, it did not improve reading or phonological scores, as shown in Table 1. Neither did it affect general cognitive abilities. Thus, neither Block Design nor Raven scores (assessing non-verbal intelligence) showed a significant improvement between the two testing sessions in the trained group $(\mathrm{p}>.11)$, though some improvement was found in the untrained group $(\mathrm{t}=3.3, \mathrm{p}=.013)$.

\section{DISCUSSION}

\section{Summary of results}

The initially poor performance of D-LDs on a range of psychoacoustic tasks could be quickly amended in the majority of practicing individuals. Learning frequency discrimination around a given reference improved performance around different reference frequencies, indicating transfer within a dimension. Moreover, with training, D-LDs gained the "anchoring benefit" which is the improvement gained in perceptual resolution when a consistent reference is used. This benefit, a robust characteristic of controls' performance (Ahissar et al., 2006), is impaired among naïve D-LDs. However, no transfer was found from pitch to duration discrimination, indicating no transfer across dimensions. Following training, D-LDs' performance on verbal working memory tasks significantly improved. Yet, pseudo-word decoding and general cognitive scores did not improve. This pattern indicates that the training program initiated specific improvement patterns. However, improvement was not specific to the inputs, but to mechanisms of working memory which seem to be shared between simple tones and complex speech sounds.

\section{Characteristics of auditory learning in $D-L D s$}

The dynamics of pattern of generalization of 2-tone discrimination tasks measured in the current study are very similar to those reported for the general population of young adults. First, 
initial learning is typically fast and substantial (e.g. Hawkey et al., 2004). Second, this learning largely (perhaps fully) transferred one octave above and one octave below the trained frequency (Demany, 1985; Irvine et al., 2000; Delhommeau et al., 2002; Ari-Even Roth et al., 2003; Amitay et al., 2005; Amitay et al., 2006). Third, in contrast to within the frequency dimension, there was no transfer of learning to duration discrimination. Fourth, learning in duration discrimination was mostly duration specific (Wright et al., 1997; Karmarkar and Buonomano, 2003), though some transfer was observed between short (100 and $200 \mathrm{~ms}$ ) tones.

These data suggest that basic learning mechanisms among teenaged D-LDs are adequate and resemble adult learning patterns rather than those characterizing younger, typically developing 6-11 year/old children. Younger children do not generalize frequency discrimination learning to novel reference frequencies nor do they generalize from reference including to no-reference protocols (Halliday et al., 2008).

\section{Transfer from tone discriminations to verbal tasks}

The transfer of learning from simple tones to speech stimuli is compelling given the specificity of learning across tone-discrimination tasks. This specificity indicates that frequency training did not result in a general "upgrade" to the auditory system of D-LDs, just as it does not do so in the general population. It further suggests that tones and verbal stimuli have shared mechanisms, presumably those related to aspects of working memory. This seems to be the case also for the general population (our lab, unpublished data). Yet, typical learning studies in the general population assess transfer across parameters and across similar paradigms, but not to general verbal memory scores. Thus, no comparison is currently available (to the best of our knowledge).

Three previous studies have shown an improvement in reading and reading related skills following perceptual training protocols in both LD (Kujala et al., 2001; Schaffler et al., 2004) and normal (Moore et al., 2005) individuals. Many differences in protocol and trained population could account for the differences in outcome between our study (finding no improvement in phonological decoding skills) and these studies. Kujala et al. (2001) trained young children who were only beginning to read and thus may have not yet acquired reading strategies that are resistant to change. Furthermore, their auditory-visual integration task may have been more 'reading related' than the tasks we used, in requiring their subjects to match auditory and visual patterns, similar to what is required when learning to read. Schaffler et al.'s (2004) study seems the most similar to ours in terms of training procedure, though they recruited dyslexic participants based on discrepancy between school grades and spelling, and therefore may have recruited a population with somewhat different characteristics than ours. Moore et al. (2005), trained typically learning children on a phoneme discrimination task in which they presented multiple phonemic contrasts in each session and found that while there was no significant learning on the trained task, trained children improved significantly more than untrained controls on phonological decoding tasks. In a fourth study (McArthur et al., 2008), poor readers who had a deficit in at-least one of four auditory tasks (frequency, vowel or consonant-vowel discrimination, or rapid auditory processing) trained on the task which posed 
the greatest difficulty for them for 6 weeks, in a manner similar to the current study. After training, most trainees had normal thresholds on that task, yet no generalization was observed to any reading related, attention or arithmetic task. Because working memory, which was the crucial aspect improved in our study, was not assessed in either of these studies, a more direct comparison between previous and current findings is not possible.

Regarding reading, perhaps the crucial parameter in transfer is the age of the trained individuals. Thus, training $8^{\text {th }}$ grade students with a paradigm aimed at basic listening skills may not solve their academic difficulties even when poor listening abilities were their main impediments at an early stage. After more than sever years in school they probably developed compensatory strategies that do not heavily rely on anchoring abilities and verbal working memory. According to this interpretation, an earlier intervention would probably have had more general effects, as is the case with other interventions for dyslexia (see Shaywitz et al., 2008).

\section{Where did learning take place?}

The fast learning rate observed in the current study makes it unlikely that the current training protocol resulted in sharpening of low level frequency or duration specific auditory representations. Instead, the outcomes of the current study are consistent with top-down driven learning mechanisms, as suggested by theories such as the Reverse Hierarchy Theory (Ahissar and Hochstein, 2004). The short training protocol probably sufficed to improve task related accessibility to lower-level representations but not their significant modification. The latter may occur with massive training protocols, as suggested by studies of highly trained musicians (Pantev et al., 1998; Wong et al., 2007). The pattern of generalization from simple auditory to verbal tasks that pound on working memory and are not initially limited by the complexity or accuracy of stimulus processing (e.g. Digit Backward) further suggests that generalization did not stem from a bottom-up progress of improved stimulus processing. Rather, the pattern of transfer suggests shared short-term memory mechanisms between simple tones and verbal sounds (see also Banai and Ahissar, 2006).

In line with the hypothesis that generally poor anchoring abilities underlie D-LDs' verbal working memory deficits (Ahissar et al., 2006; Ahissar, 2007) improvement in anchoring ability occurred concurrently with improvement in verbal memory. The pattern of no generalization from frequency to duration and yet substantial generalization to verbal stimuli after having learned both is intriguing. Current data allows several interpretations. The effect of improving anchoring abilities is perhaps gradually generalized with training and assessments on additional parameters and dimensions. Perhaps the current training protocol provided the opportunity to anchor to a repeated reference, and access its lower-level representations on the one hand, and yet allowed generalization by introducing novel conditions when significant learning occurred.

Taken together, the main novelty of this study is pointing to shared mechanisms of implicit memory across simple and complex stimuli. This common bottleneck on performance of seemingly low and high level tasks, introduces the potential of using perceptual training protocols as a tool to boost cognitive skills in various populations. 


\section{ACKNOWLEDGEMENTS}

This work was supported by the Israel Science Foundation, by the Israeli Institute for Psychobiology And by a subcontract NIH grant. We thank all participants of this study.

\section{REFERENCES}

Ahissar, M. (2007): Dyslexia and the anchoring-deficit hypothesis. Trends Cogn. Sci., 11, 458-465.

Ahissar, M., Hochstein, S. (1997): Task difficulty and the specificity of perceptual learning. Nature, 387, 401-406.

Ahissar, M., Hochstein, S. (2004): The reverse hierarchy theory of visual perceptual learning. Trends Cogn. Sci., 8, 457-464.

Ahissar, M., Protopapas, A., Reid, M., Merzenich, M. M. (2000): Auditory processing parallels reading abilities in adults. Proc Natl. Acad. Sci. USA, 97, 6832-6837.

Ahissar, M., Lubin, Y., Putter-Katz, H., Banai, K. (2006): Dyslexia and the failure to form a perceptual anchor. Nat. Neurosci., 9, 1558-1564.

Amitay, S., Ahissar, M., Nelken, I. (2002a): Auditory processing deficits in reading disabled adults. $J$. Assoc. Res. Otolaryngol., 3, 302-320.

Amitay, S., Hawkey, D. J., Moore, D. R. (2005): Auditory frequency discrimination learning is affected by stimulus variability. Percept. Psychophys., 67, 691-698.

Amitay, S., Irwin, A., Moore, D. R. (2006): Discrimination learning induced by training with identical stimuli. Nat. Neurosci., 9, 1446-1448.

Amitay, S., Ben-Yehudah, G., Banai, K., Ahissar, M. (2002b): Disabled readers suffer from visual and auditory impairments but not from a specific magnocellular deficit. Brain, 125, 2272-2285.

Ari-Even Roth, D., Amir, O., Alaluf, L., Buchsenspanner, S., Kishon-Rabin, L. (2003): The effect of training on frequency discrimination: Generalization to untrained frequencies and to the untrained ear. J. Basic Clin. Physiol. Pharmacol., 14, 137-150.

Banai, K., Ahissar, M. (2004): Poor frequency discrimination probes dyslexics with particularly impaired working memory. Audiol. Neurootol., 9, 328-340.

Banai, K., Ahissar, M. (2006): Auditory processing deficits in dyslexia: Task or stimulus related? Cerebral Cortex, 16, 1718-1728.

Ben-Yehudah, G., Banai, K., Ahissar, M. (2004): Patterns of deficit in auditory temporal processing among dyslexic adults. Neuroreport, 15, 627-631.

Ben-Yehudah, G., Sackett, E., Malchi-Ginzberg, L., Ahissar, M. (2001): Impaired temporal contrast sensitivity in dyslexics is specific to retain-and-compare paradigms. Brain, 124, 1381-1395.

De Weirdt, W. (1988): Speech perception and frequency discrimination in good and poor readers. Appl. Psycholinguistics, 9, 163-183.

Delhommeau, K., Micheyl, C., Jouvent, R., Collet, L. (2002): Transfer of learning across durations and ears in auditory frequency discrimination. Percept. Psychophys., 64, 426-436.

Demany, L. (1985): Perceptual learning in frequency discrimination. J. Acoust. Soc. Am., 78, 1118-1120.

Deutsch, A., Bentin, S. (1996): Attention factors mediating syntactic deficiency in reading-disabled children. J. Exp. Child Psychol., 63, 386-415.

Everatt, J., Weeks, S., Brooks, P. (2008): Profiles of strengths and weaknesses in dyslexia and other learning difficulties. Dyslexia, 14, 16-41.

Fahle, M. (2005): Perceptual learning: Specificity versus generalization. Curr. Opin. Neurobiol., 15, $154-160$.

Learning \& Perception 1 (2009) 1 
Fahle, M., Poggio, T. (2002): Perceptual Learning. Cambridge, MA: MIT Press.

Gathercole, S. E., Alloway, T. P., Willis, C., Adams, A. M. (2006): Working memory in children with reading disabilities. J. Exp. Child Psychol., 93, 265-281.

Goldstone, R. L. (1998): Perceptual learning. Annu. Rev. Psychol., 49, 585-612.

Halliday, L. F., Taylor, J. L., Edmondson-Jones, A. M., Moore, D. R. (2008): Frequency discrimination learning in children. J. Acoust. Soc. Am., 123, 4393-4402.

Hari, R., Renvall, H. (2001): Impaired processing of rapid stimulus sequences in dyslexia. Trends in Cognitive Sciences, 5, 525-532.

Heath, S. M., Hogben, J. H., Clark, C. D. (1999): Auditory temporal processing in disabled readers with and without oral language delay. J. Child Psychol. Psychiatry, 40, 637-647.

Irvine, D. R., Martin, R. L., Klimkeit, E., Smith, R.. (2000): Specificity of perceptual learning in a frequency discrimination task. J. Acoust. Soc. Am., 108, 2964-2968.

Karmarkar, U. R., Buonomano, D. V. (2003): Temporal specificity of perceptual learning in an auditory discrimination task. Learn. Mem., 10, 141-147.

Kujala, T., Karma, K., Ceponiene, R., Belitz, S., Turkkila, P., Tervaniemi, M., Naatanen, R. (2001): Plastic neural changes and reading improvement caused by audiovisual training in reading-impaired children. Proc. Natl. Acad. Sci. USA, 98, 10509-10514.

McAnally, K. I., Stein, J. F. (1996): Auditory temporal coding in dyslexia. Proc. R. Soc. Lond. B Biol. Sci., 263, 961-965.

McArthur, G. M., Bishop, D. V. (2001): Auditory perceptual processing in people with reading and oral language impairments: Current issues and recommendations. Dyslexia, 7, 150-170.

McArthur, G. M., Ellis, D., Atkinson, C. M., Coltheart, M. (2008): Auditory processing deficits in children with reading and language impairments: Can they (and should they): be treated? Cognition, 107, 946-977.

Moore, D. R., Rosenberg, J. F., Coleman, J. S. (2005): Discrimination training of phonemic contrasts enhances phonological processing in mainstream school children. Brain Lang., 94, 72-85.

Nahum, M., Nelken, I., Ahissar, M. (2008): Low-level information and high-level perception: The case of speech in noise. PLoS Biol., 6, e126.

Pantev, C., Oostenveld, R., Engelien, A., Ross, B., Roberts, L. E., Hoke, M. (1998): Increased auditory cortical representation in musicians. Nature, 392, 811-814.

Raven, J., Raven, J. C., Court, J. H. (2000): Manual for Raven's Progressive Matrices and Vocabulary Scales. Oxford: Oxford Psychologists Press.

Reed, M. A. (1989): Speech perception and the discrimination of brief auditory cues in reading disabled children. J. Exp. Child Psychol., 48, 270-292.

Roach, N. W., Hogben, J. H. (2008): Spatial cueing deficits in dyslexia reflect generalised difficulties with attentional selection. Vision Res., 48, 193-207.

Schaffler, T., Sonntag, J., Hartnegg, K., Fischer, B. (2004): The effect of practice on low-level auditory discrimination, phonological skills, and spelling in dyslexia. Dyslexia, 10, 119-130.

Sharma, M., Purdy, S. C., Newall, P., Wheldall, K., Beaman, R., Dillon, H. (2006): Electrophysiological and behavioral evidence of auditory processing deficits in children with reading disorder. Clin. Neurophysiol., 117, 1130-1144.

Shaywitz, S. E., Morris, R., Shaywitz, B. A. (2008): The education of dyslexic children from childhood to young adulthood. Annu. Rev. Psychol., 59, 451-475.

Snow, C. E., Burns, M. S., Griffin, P. (eds) (1998): Preventing Reading Difficulties in Young Children. Washington, D.C.: National Academic Press.

Swanson, H. L. (1993): Working memory in learning disability subgroups. J. Exp. Child Psychol., 56, $87-114$.

Swanson, H. L., Sachse-Lee, C. (2001): A subgroup analysis of working memory in children with reading disabilities: Domain-general or domain-specific deficiency? J. Learn. Disabil., 34, 249-263. 
Tallal, P. (1980): Auditory temporal perception, phonics, and reading disabilities in children. Brain Lang., 9, 182-198.

Torgesen, J. K. (1988): Studies of children with learning disabilities who perform poorly on memory span tasks. J. Learn. Disabil., 21, 605-612.

Wechsler, D. (1998): Wechsler Intelligence Scale for Children (R-95,1998 Israeli edition): - Manual, R-95 1998. Israeli edition. San Antonio: The Psychological Corporation.

Willcutt, E. G., Pennington, B. F. (2000): Comorbidity of reading disability and attention-deficit/hyperactivity disorder: Differences by gender and subtype. J. Learn. Disabil., 33, 179-191.

Witton, C., Stein, J. F., Stoodley, C. J., Rosner, B. S., Talcott, J. B. (2002): Separate influences of acoustic AM and FM sensitivity on the phonological decoding skills of impaired and normal readers. $J$. Cogn. Neurosci., 14, 866-874.

Wong, P. C., Skoe, E., Russo, N. M., Dees, T., Kraus, N. (2007): Musical experience shapes human brainstem encoding of linguistic pitch patterns. Nat. Neurosci., 10, 420-422.

Wright, B. A., Buonomano, D. V., Mahncke, H. W., Merzenich, M. M. (1997): Learning and generalization of auditory temporal-interval discrimination in humans. J. Neurosci., 17, 3956-3963. 